\title{
Building a Classification Model to Predict School Quality in Indonesia
}

\author{
Satriyo Wibowo* \\ Directorate General of Early Childhood Education, Primary Education, and Secondary Education \\ Ministry of Education and Culture \\ Jakarta, Indonesia \\ *satriyo.wibowo@kemdikbud.go.id
}

\begin{abstract}
The aim of the present study was to explore the best classification model based on the predictive performance of decision tree (DT), random forest (RF), and boosted tree (BT) models used for school quality status detection in Indonesia. In order to get a better insight into the predictive abilities of these three models, 213,536 records from Basic Education Data (Dapodik), an education management information system managed by Ministry of Education and Culture, were utilized. A total of 23 predictors were extracted from Dapodik. School quality status (two classes: 'met national education standards' and 'improvement required') was an output (dependent) variable. Accuracy of the DT, RF, and BT models evaluated on the training dataset and the testing dataset was $71.51 \%, 99.91 \%$, and $72.66 \%$ as well as $71.53 \%, 73.56 \%$, and $72.82 \%$, respectively. The findings indicate that the BT model had better performance than the DT dan RF models.
\end{abstract}

Keywords—school quality, decision tree, random forest, boosted tree, classification

\section{INTRODUCTION}

Ministry of Education and Culture has developed an education management information system called Basic Education Data (Dapodik) which collects data from schools on school facilities, students, teachers and education personnel, and educational substances. Dapodik as the only data collecting system is continuously updated each semester and has been widely used in assessing the quality of schools.

Many researchers have studied the effect of school quality using measures such as school leadership and management, student and teacher characteristics, school facilities, and community involvement on student outcomes [1-3]. The study of school quality and factors associated with are growing fields of research in the education sciences. Furthermore, extensive progress has been made in the development of algorithms for modeling and identifying information from big databases. This study proposes data mining techniques to identify the key factors that classify and differentiate high- and low-quality schools.

Classification modeling is one of the analytical tools that can be utilized to distinguish the most influential factors.
Classification is an operation that places each object from the population in one of a number of specified classes, according to the characteristics of the object which are identified as independent variables. An object is generally assigned to a class by using a formula, an algorithm, or a set of rules, which forms a model [4]. Classification modeling can be used to build predictive models and identify factors that affect school quality. Previous studies use classification modeling such as decision tree (DT), random forest (RF), and boosted tree (BT) to identify main factors of school accreditation rank and quality of education $[5,6]$.

The DT model is a type of supervised learning algorithm (having a pre-defined target variable) that is mostly used in classification researches. The DT model uses the C4.5 algorithm to form a decision tree. The trees are generated when the response variable is categorical data [7] This C4.5 algorithm is a development of the earlier ID3 algorithm and has the advantage of being able to process numeric and discrete data and can handle missing attribute values. In the decision tree, each leaf node is assigned as a class label. The root node and internal node contain attribute test conditions to separate records that have different characteristics [8].

The RF model is a type of ensemble machine learning algorithm called bootstrap aggregating (bagging) method and random feature selection [9]. Unlike the classification and regression tree (CART) method, the RF model is a compound tree method. In the RF model, many classification trees are built to form a forest, then the analysis is carried out based on voting from the tree clusters of the classification.

Meanwhile, the BT model is an iterative algorithm that also uses a combination of several classification trees that give different weights to the distribution of training data for each iteration. Each boosting iteration adds weight to the examples of misclassification and reduces the weight to the correct classification example so that it can effectively change the distribution of training data [10]. The adaptive boosting method (AdaBoost) can be a more effective solution to class imbalance problems [11]. In this study, 3 methods of classification modeling (DT, RF, and BT models) were 
analyzed to find the most accurate model to predict school quality status.

\section{METHODS}

\section{A. Data}

School quality status and Basic Education Data (Dapodik) datasets were used in this study. The school quality status dataset was extracted from the 2019 school quality index generated by the Center of Data and Statistics for Education and Culture, Ministry of Education and Culture in building a zoning-based education quality index [12]. The school quality status can be categorized into two classes namely 'met national education standards' (class ' 1 ') and 'improvement required' (class '0').

TABLE I. LIST OF PREDICTORS

\begin{tabular}{|l|l|}
\hline $\begin{array}{c}\text { Variable } \\
\text { Name }\end{array}$ & \\
\hline X1 & School has headmaster \\
\hline X2 & School has headmaster with bachelor degree \\
\hline X3 & School has certified headmaster \\
\hline X4 & School has trained headmaster \\
\hline X5 & School-based management applied \\
\hline X6 & School operational assistance fund program (BOS) allocated \\
\hline X7 & School operating time \\
\hline X8 & School has ISO certification \\
\hline X9 & School has accreditation rank \\
\hline X10 & Student teacher ratio \\
\hline X11 & Student class groups ratio \\
\hline X12 & Student classroom ratio \\
\hline X13 & Percentage of teachers with bachelor degree \\
\hline X14 & Percentage of certified teachers \\
\hline X15 & $\begin{array}{l}\text { Number of proposed students to get Smart Indonesia } \\
\text { Program (PIP) }\end{array}$ \\
\hline X16 & Number of dropout students \\
\hline X17 & School ownership status \\
\hline X18 & Source of electricity \\
\hline X19 & School has internet connection \\
\hline X20 & Number of good condition classrooms \\
\hline X21 & School has library \\
\hline X22 & Number of computers \\
\hline X23 & School has parent teacher committee \\
\hline
\end{tabular}

A total of 23 variables were generated and used as predictors to classify school quality status. The list of those predictors is shown in Table 1. An initial dataset contained 215,866 schools extracted from Dapodik but was subsequently reduced after editing for missing, incorrect values and outliers. The final dataset had 213,536 schools and will be used to build the model. As seen in Table 2, the data consists of two classes of school quality status namely 'met national education standards' and 'improvement required'.

TABLE II. DistribUtion OF SCHOOLS By QUALITY STATUS

\begin{tabular}{|l|r|r|}
\hline \multicolumn{1}{|c|}{ Status } & Frequency & Percent \\
\hline Met national education standards & 65,104 & $30.5 \%$ \\
\hline Improvement required & 148,432 & $69.5 \%$ \\
\hline Total & 213,536 & $100.0 \%$ \\
\hline
\end{tabular}

\section{B. Method}

In this paper, the analysis was carried out using $\mathrm{R}$ software by comparing the performance of DT, RF, and BT models as follows:

- Splitting the data into a training dataset and testing dataset (70\% vs 30\%)

- Create DT, RF, and BT models using the training dataset

- Evaluate the predictive performance of the DT, RF, and BT models. The difference between predicted and expected binary school quality status was described by the number of True Positives (TP), True Negatives $(T N)$, False Positives $(F P)$, False Negatives $(F N)$, where the sum of $T P+T N+F P+F N=n$ is the total number of schools. To assess the model predictive accuracy of DT, RF and BT model for school quality status, the following performance metrics were used: correct classification rate $(C=(T P+T N) / n), \quad$ sensitivity $\left(S_{n}=T P /(T P+F N)\right), \quad$ specificity $\quad\left(S_{p}=T N /(T N+F P)\right)$, positive predictive value $(P P V=T P /(T P+F P))$, negative predictive value $(N P V=T N /(T N+F N))$. Moreover, to illustrate the relationship between sensitivity and specificity for the two-class category, the receiver operating characteristic (ROC) curve was plotted and the area under the ROC curves $(A U C)$ was calculated [13].

\section{RESULTS AND DISCUSSION}

The confusion matrices from the DT model is shown in Fig. 1. The DT model was able to correctly predict school quality status in $\mathrm{C}=71.51 \%$ for the training dataset and $\mathrm{C}=71.53 \%$ for the testing dataset (Fig. 1). The results indicate that the DT model provided a fairly stable prediction and no overfitting occurred. Fig. 2 shows the classifier ROC curves with the AUC $=0.6264$ on training samples and AUC $=0.6245$ for the DT prediction on test samples. When evaluated, the DT model has a specificity of $88.34 \%$ for the training dataset and $88.01 \%$ for testing dataset which means it is good at predicting 'improvement required' schools.

A RF of 100 trees achieved $\mathrm{C}=99.91 \%$ during the training phase and correctly classified $\mathrm{C}=73.56 \%$ of the testing dataset. The results indicate that the RF model provided unstable prediction and overfitting occurred. The confusion matrices from the RF model is shown in Fig. 3. Fig. 4 shows the ROC curve with the AUC $=0.9996$ for RF prediction on the training dataset and $\mathrm{AUC}=0.7508$ on the testing dataset. When evaluated, the RF model has a specificity of $99.98 \%$ for the training dataset and $89.89 \%$ for the testing dataset. This means that the RF model is good at predicting poor schools.

The BT model provided an accuracy rate of $\mathrm{C}=72.66 \%$ for the training dataset and $\mathrm{C}=72.82 \%$ for the testing dataset. The results indicate that the $\mathrm{BT}$ model provided a fairly stable prediction and no overfitting occurred. The confusion matrices of the BT model are shown in Fig. 5 while Fig. 6 shows the 
ROC curve with the AUC $=0.7423$ on training samples and $\mathrm{AUC}=0.7390$ on test samples. When further evaluated, the BT model has a specificity of $92.93 \%$ for the training dataset and $92.74 \%$ for the testing dataset which higher than those DT and RF models. This BT model is the best for predicting non-good performing schools.

DT Training Confusion Matrix

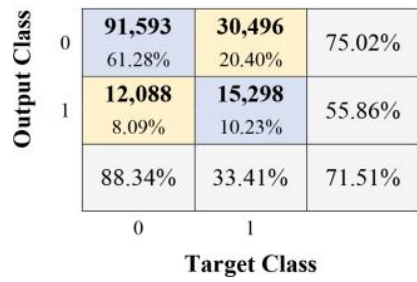

Fig. 1. Confusion matrices for the training dataset (left) and the test samples (right) for DT model.
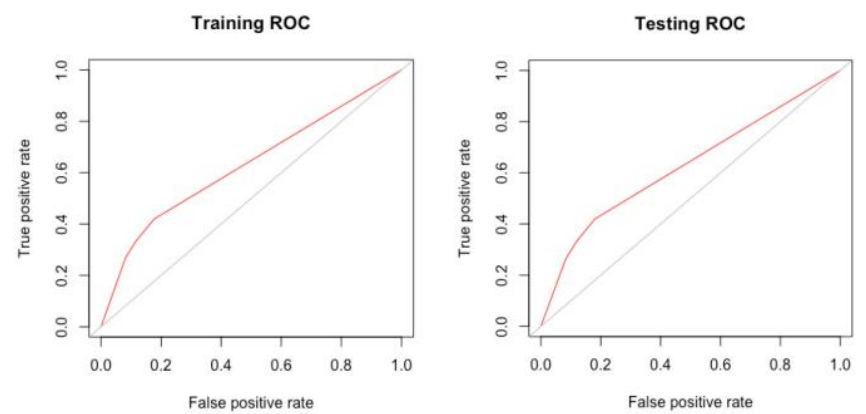

Fig. 2. ROC curves for DT classification accuracy on the training (left) and test (right) samples matrices for the training dataset (left) and the test samples (right) for DT model.

\section{RF Training Confusion Matrix}

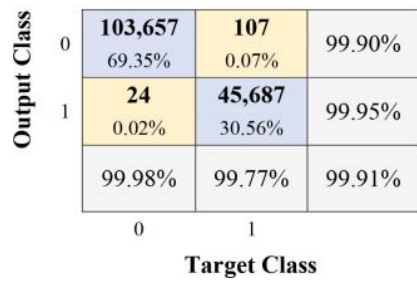

RF Testing Confusion Matrix

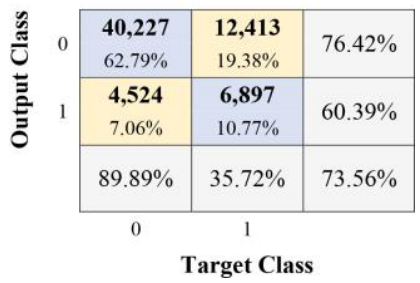

Fig. 3. Confusion matrices for the training dataset (left) and the test samples (right) for RF model.

TABLE III. Predictive Performance of The Three Machine LeARning Models

\begin{tabular}{|c|c|c|c|c|c|c|}
\hline \multirow{2}{*}{ Performance } & \multicolumn{2}{|c|}{ DT } & \multicolumn{2}{|c|}{ RF } & \multicolumn{2}{|c|}{ Boosting } \\
\hline & Training & Testing & Training & Testing & Training & Testing \\
\hline Correct classification rate, $\mathrm{C}(\%)$ & $71.51 \%$ & $71.53 \%$ & $99.91 \%$ & $73.56 \%$ & $72.66 \%$ & $72.82 \%$ \\
\hline Sensitivity, Sn (\%) & $33.41 \%$ & $33.34 \%$ & $99.77 \%$ & $35.72 \%$ & $26.75 \%$ & $26.66 \%$ \\
\hline Specificity, Sp (\%) & $88.34 \%$ & $88.01 \%$ & $99.98 \%$ & $89.89 \%$ & $92.93 \%$ & $92.74 \%$ \\
\hline Positive Predictive Value, PPV (\%) & $55.86 \%$ & $54.54 \%$ & $99.95 \%$ & $60.39 \%$ & $62.58 \%$ & $61.29 \%$ \\
\hline Negative Predictive Value, NPV (\%) & $75.02 \%$ & $75.37 \%$ & $99.90 \%$ & $76.42 \%$ & $74.18 \%$ & $74.56 \%$ \\
\hline Area under the ROC curve, AUC & 0.6264 & 0.6245 & 0.9996 & 0.7508 & 0.7423 & 0.7390 \\
\hline
\end{tabular}

Fig. 4. ROC curves for RF classification accuracy on the training (left) and test (right) samples matrices for the training dataset (left) and the test samples (right) for RF model.

BT Training Confusion Matrix

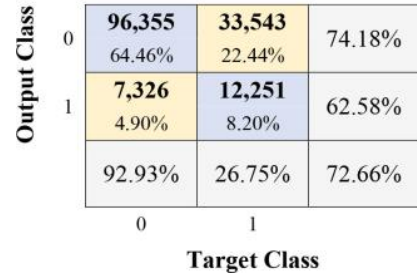

Fig. 5. Confusion matrices for the training dataset (left) and the test samples (right) for BT model.

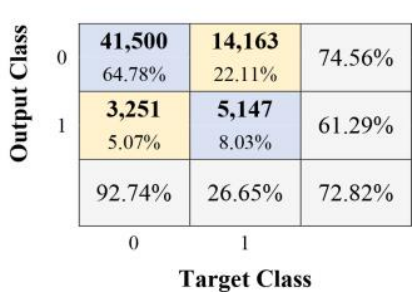

Target Clas

Based on the findings of this study, the RF model gave an verfitting result. The RF model was poorly performed in higher accuracy than the DT model. In addition, the BT model and Ahmed [14] that concluded Decision Tree model shows provides better AUC score than the Decision Tree in predicting health expenditures.
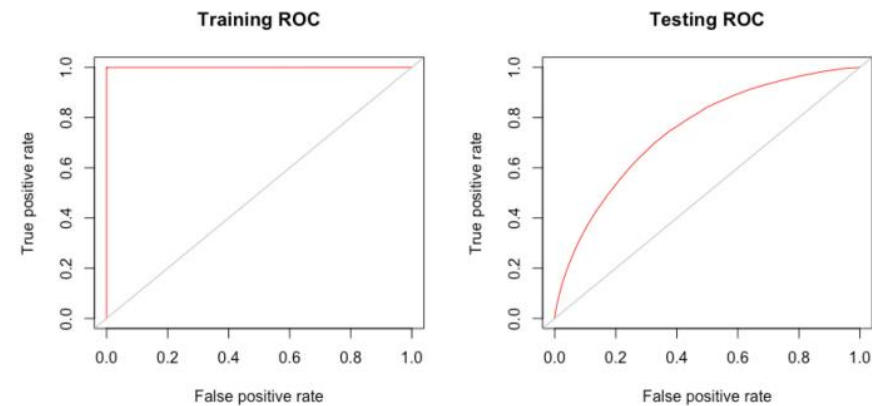
4 
Training ROC

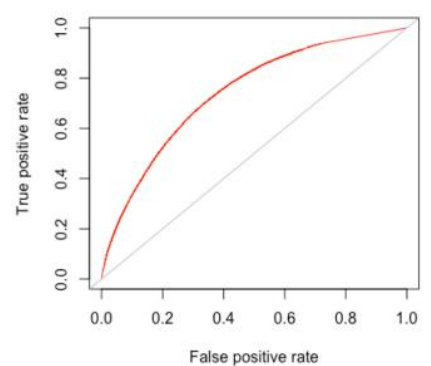

Testing ROC

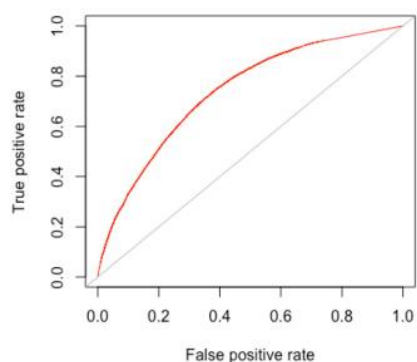

Fig. 6. ROC curves for BT classification accuracy on the training (left) and test (right) samples matrices for the training dataset (left) and the test samples (right) for BT model.

The findings about BT model performance in this study was supported from the research conducted by Esseiva et al. [16] that examined the classification modeling on feet fidgeting detection based on accelerometers and concluded that the Boosting model provides better accuracy than the Decision Tree and Random Forest. Miao and Heaton [17] study also showed that Boosting provides higher classification accuracy than Random Forest in predicting ecosystem classification data in the east Mojave Desert (see table 3).

\section{CONCLUSION}

This paper presents preliminary research and describes the importance of tree-based machine learning algorithm and their application in predicting school quality status. The results show that the BT model emerged best compared to DT and RF models. The proposed tree-based algorithms have limitations. They require correct parameter tuning, the BT model may be overfitting if imprecise parameters are used. The existing dataset didn't have most of the school features such as learning materials, laboratories, and academic performance. In the future, we consider expanding the scope of Dapodik utilization to extract insights that may be valuable to school stakeholders.

\section{ACKNOWLEDGMENT}

This work was supported by the Secretariat of Directorate General of Early Childhood Education, Primary Education, and Secondary Education.

\section{REFERENCES}

[1] M. Shodiq, Suyata, and S. Wibawa, "Developing quality evaluation instrument for islamic senior high school," Jurnal Penelitian dan Evaluasi Pendidikan, vol. 21, no. 2, pp. 189-205, December 2017.

[2] S. Wu, C. Lin, S. Wu, C. Chuang, and H. Kuan, "Factors affecting quality of service in schools in Hualien, Taiwan," in 5th World Conference on Educational Sciences (WCES), 2013, pp. 1160-1164.

[3] D.D. Goldhaber and D.J. Brewer, "Does teacher certification matter? High school teacher certification status and student achievement," Educ. Eval. Policy Anal, vol. 22, no. 2, pp. 129-145, 2000.

[4] S. Tufféry, Data Mining and Statistics for Decision Making. West Sussex: John Wiley \& Sons Ltd, 2011.

[5] Y. Nindahayati, "Building a model to predict school accreditation rank using boosted classification tree," Master's thesis (unpublished), IPB University, 2015.

[6] A. Ramadhan, B. Susetyo, and Indahwati, "Penerapan metode klasifikasi random forest dalam mengidentifikasi faktor penting penilaian mutu pendidikan," Jurnal Pendidikan dan Kebudayaan, vol. 4, no. 2, pp. 169 182, December 2019.

[7] L. Breiman, J.H. Friedman, R.A. Olshen, and C.J. Olsen, Classification and Regression Trees. Boca Raton: Chapman \& Hall, 1984.

[8] P. Tan, M. Steinbach, and V. Kumar, Introduction to Data Mining. Essex: Pearson Education Limited, 2014.

[9] L. Breiman, "Random forests," Machine Learning, Vol. 45, pp. 5-32, October 2001

[10] S. Kotsiantis, D. Kanellopoulos, and P. Pintelas, "Handling imbalanced datasets: a review," GESTS International Transactions on Computer Science and Engineering, vol. 30, pp. 25-36, 2006.

[11] S.B. Kotsiantis and P.E. Pintelas, "Selective costing ensemble for handling imbalanced data sets," International Journal of Hybrid Intelligent System, vol. 6, pp. 123-133, 2009.

[12] Ministry of Education and Culture, Indeks Mutu Pendidikan Berbasis Zonasi. Jakarta: PDSPK, 2019.

[13] S. Rogers and M. Girolami, A First Course in Machine Learning, 2nd edition. Boca Raton: CRC Press, 2017.

[14] M.H. Sadiq and N.S. Ahmed, "Classifying and predicting students' performance using improved decision tree C4.5 in higher education institutes," Journal of Computer Science, vol. 15, no. 9, pp. 1291-1306, October 2019.

[15] S. Cinaroglu, "Comparison of performance of decision tree algorithms and random forest: an application on OECD countries health expenditures," International Journal of Computer Applications, vol. 138, no. 1, pp. 37-41, March 2016.

[16] J. Esseiva, M. Caon, E. Mugellini, O.A. Khaled, and K. Aminian, "Feet Fidgeting Detection Based on Accelerometers Using Decision Tree Learning and Gradient Boosting," In: I. Rojas and F. Ortuño (Eds.) IWBBIO 2018. Lecture Notes in Computer Science, vol. 10814, pp. 7584, 2018.

[17] X. Miao and J.S. Heaton, "A comparison of random forest and Adaboost tree in ecosystem classification in east Mojave Desert," 2010 18th International Conference on Geoinformatics, pp. 1-6, 2010. 